\title{
Top-down and bottom-up attentional control: On the nature of interference from a salient distractor
}

\author{
MIN-SHIK KIM \\ Yonsei University, Seoul, Korea \\ and \\ KYLE R. CAVE \\ University of Southampton, Southampton, England
}

\begin{abstract}
In two experiments using spatial probes, we measured the temporal and spatial interactions between top-down control of attention and bottom-up interference from a salient distractor in visual search. The subjects searched for a square among circles, ignoring color. Probe response times showed that a color singleton distractor could draw attention to its location in the early stage of visual processing (before a 100-msec stimulus onset asynchrony [SOA]), but only when the color singleton distractor was located far from the target. Apparently the bottom-up activation of the singleton distractor's location is affected early on by local interactions with nearby stimulus locations. Moreover, probe results showed that a singleton distractor did not receive attention after extended practice. These results suggest that top-down control of attention is possible at an early stage of visual processing. In the long-SOA condition (150-msec SOA), spatial attention selected the target location over distractor locations, and this tendency occurred with or without extended practice.
\end{abstract}

One of the important questions in attention is how we selectively process information that is relevant to the current task, while ignoring information that is irrelevant to the task. Many studies have used the visual search paradigm to investigate this selection process. In general, they show that when the interfering distractors share more features with the target, they make the search more difficult (Duncan \& Humphreys, 1989; Treisman \& Gelade, 1980; Wolfe, Cave, \& Franzel, 1989). On the other hand, a distractor with a different feature from the target and the other distractors can also make search more difficult (Pashler, 1988; Theeuwes, 1991,1992).

These seemingly incompatible phenomena can be explained by two distinct components of attentional control, typically referred to as bottom-up and top-down control (Bundesen, 1990; Cave \& Wolfe, 1990; Duncan \& Humphreys, 1989; Eriksen \& Hoffman, 1972; Koch \& Ullman, 1985; Posner, 1980; Treisman \& Sato, 1990; Yantis $\&$ Jonides, 1984). The former has also been referred

Data from Experiment 1 were presented at the Annual Meeting of the Association for Research in Vision and Ophthalmology (ARVO), April, 1996, Fort Lauderdale, FL. This work was supported in part by NEI Grant EY 08126 to the Vanderbilt Vision Research Center. Thanks to Amy Coombs for help in testing subjects and to Narcisse Bichot, Randolph Blake, Keith Clayton, Joe Lappin, Alan Peters, and Jeff Schall for helpful suggestions. Correspondence concerning this article should be addressed to either M.-S. Kim, Department of Psychology, Yonsei University, Seoul, 120-749. Korea, or K. R. Cave, Department of Psychology, University of Southampton, Highfield, Southampton SO17 1BJ, United Kingdom (e-mail: coglit.soton.ac.uk).

-Accepted by previous editor. Myron L. Braunstein to as stimulus-driven or exogenous control and is said to be based on the properties of the stimulus, irrespective of our advance knowledge of the stimulus or of our intentions or goals to an attentional task. The latter has been referred to as goal-directed or endogenous control and is concerned with how our advance knowledge, intentions, and goals control visual selection. Much research has been performed to investigate how these two distinct modes of attentional control influence attentional performance.

In many search models, bottom-up activation is generally emphasized in preattentive parallel search, such as simple feature search, in which feature singletons pop out. In attentive, serial search, such as complex conjunction search, however, top-down activation that relies on the knowledge of the target features plays a critical role in guiding attention. However, few studies have shown how these two attentional factors develop over time or how they change after extended practice.

In the cuing paradigm, both central (indirect) cues and peripheral (direct) cues have been used to understand mechanisms underlying top-down and bottom-up control of attentional selection. In many early studies, a central cue took the form of an arrow presented at the center of the screen, indicating the likely target position, whereas a peripheral cue took the form of a stimulus presented at or near the indicated location. When the cue was valid (indicating the target location correctly) and had some predictive value, it typically resulted in benefits in responding to the target, independently of the cue type (see, e.g., Jonides, 1981; Posner, 1980; Posner, Snyder, \& Davidson, 1980). On the other hand, a clear distinction between central and peripheral cues arose when they had 
no predictive value. When subjects knew that the cue location was irrelevant to the target location, they could easily ignore central cues, but not peripheral cues (see, e.g., Jonides, 1981; Müller \& Rabbitt, 1989). Moreover, even when subjects knew that peripheral cues were always invalid, they could not ignore the cues (Remington, Johnston, \& Yantis, 1992). These results suggest two functionally separable mechanisms of attentional control.

Recently, several studies further examined these two separable modes of attention and showed that they have relatively different time courses (e.g., Cheal \& Lyon, 1991; Nakayama \& Mackeben, 1989; Yantis \& Jonides, 1990). In general, these studies suggest that the top-down (or sustained) component of attention slowly activates a location indicated by a central cue and remains there in a steady fashion, whereas the bottom-up (or transient) component activates the peripherally cued location rapidly and dissipates within a few hundred milliseconds. Although many current models of attention generally agree that attentional deployment results from an interaction between the top-down and the bottom-up components, there is still considerable debate regarding the extent to which the goal-directed, task-relevant top-down control of attention can overcome the bottom-up activation of task-irrelevant stimuli from the early visual processing. The main question is, when a distractor is very salient and thus produces strong bottom-up activation, how effectively and how quickly can top-down attention suppress the task-irrelevant bottom-up activation (see Bacon \& Egeth, 1994; Theeuwes, 1991, 1992, 1994).

One group of researchers has demonstrated conditions under which a salient feature singleton does not capture attention when it is irrelevant to the task, implying that the top-down control of attention is overriding the bottomup activities (Folk \& Annett, 1994; Hillstrom \& Yantis, 1994; Jonides \& Yantis, 1988; Koshino, Warner, \& Juola, 1992; Yantis \& Egeth, 1994; Yantis \& Jonides, 1990). In their studies, subjects usually focused their attention on a restricted area indicated in advance by a central cue. Another group of researchers has shown that task-irrelevant feature singletons can capture attention in a bottom-up fashion (Pashler, 1988; Theeuwes, 1991, 1992) in other situations. For example, Theeuwes $(1991,1992)$ performed visual search experiments with a simple feature target. Among the many distractors, there was one distractor with a salient unique feature in a task-irrelevant dimension. He found that the irrelevant salient distractor interfered with parallel "preattentive" search for a feature target. A recent study by Theeuwes (1995a) also suggested that the interference from the singleton distractor is based on spatial selection at that location, rather than on nonspatial attention.

Theeuwes (1992, 1994, 1995a) provided an explanation for the conflicting results. According to his interpretation, when subjects search for a target among many distractors and thus "divide" attention over the visual field, a task-irrelevant featural singleton can capture attention. However, when subjects focus their attention on a specific region before display onset, as in the cuing procedures, they do not engage in parallel preattentive search and, thus, distraction from a singleton feature does not occur (but see Bacon \& Egeth, 1994; Folk, Remington, \& Johnston, 1992; Folk, Remington, \& Wright, 1994).

In a more recent experiment, Theeuwes (1995b) presented a task-irrelevant abrupt onset at one of three different distances from the target location. The spatial distracting effect from the abrupt onset occurred when the stimulus onset asynchrony (SOA) between the search display and the abrupt onset was less than $100 \mathrm{msec}$. After $100 \mathrm{msec}$, however, the abrupt onset did not interfere with the search for the feature target. Theeuwes interpreted these results as supporting two functionally different processes: an early preattentive processing that allows the parallel detection of any salient features on the basis of bottom-up activation of visual stimuli and then an attentive processing in which attention is focused on a restricted area.

Theeuwes concluded that the preattentive stage of processing is driven only by bottom-up factors (Theeuwes, 1991, 1992, 1994, 1995b). However, note that the response time (RT) interference that he used in his studies does not necessarily indicate that the top-down control of attention is completely absent in preattentive processing. In his studies, for example, when there is a task-irrelevant singleton distractor, both the target and the singleton distractor locations might be simultaneously competing for selection, and top-down control might operate successfully to select the target location on some trials, but not on other trials, owing to high levels of noise. Or, at the preattentive level, the top-down control of attention might partially inhibit the bottom-up activation from a taskirrelevant salient distractor. These alternative interpretations, in which there is still top-down control at the preattentive level, can explain the slower RTs in the singleton distractor condition, as compared with the no-singleton distractor condition, in Theeuwes's studies. These increased RTs do not necessarily mean that the target location does not receive any spatial attention guided by topdown selectivity.

Although Theeuwes (1995a) showed evidence for spatial distraction caused by a singleton distractor, it is still unclear whether the target location also has more or less the same amount of spatial attention as does the singleton distractor location. Therefore, it is important to measure how the strength of spatial attention varies at the target and distractor singleton locations at different points in time. Measuring spatial attention at the location of each of the search elements will, in turn, help us to understand how both bottom-up and top-down components of attention develop over time. For testing these issues, one reasonable method would be to use a dual-task procedure with postdisplay probes.

Our earlier studies combined spatial probes with visual search tasks (Bichot, Cave, \& Pashler, 1999; Cave \& Zimmerman, 1997; Cepeda, Cave, Bichot, \& Kim, 1998; Kim \& Cave, 1995, 1999, in press). The primary task was 
to search for a predefined target among many distractors. The subjects held their response to the search until prompted for it by the computer. This delay allowed time for the probe stimulus, a small black dot, to appear on some trials. The probe appeared at one of the search element locations equally often, regardless of the target location. In response, the subjects pressed a button as quickly as possible. Also, the probes appeared with variation in SOA, allowing the investigation of attentional changes over time. In Kim and Cave's (1995) study, for example, the subjects in conjunction search showed faster responses to probes at the target location and, also, to probes at the locations containing a distractor with the target color or the target shape, indicating that spatial attention selects the locations with the target features on the basis of top-down knowledge.

We also measured spatial attention in feature searches and found that, in some circumstances, spatial attention selected the location occupied by a simple feature target that had a unique feature in the stimulus array. Although that study showed that spatial attention is allocated to the location of the easily discriminable feature target, it could not determine the relative contributions of top-down and bottom-up factors to this pattern of attention. In this simple visual search task, the target has a singleton feature. Attention can be directed to the location of the target by a top-down selectivity based on a known-to-be-relevant target feature, by a bottom-up activation from the unique feature of the target, or by both.

The present study will use simple feature search as a primary task in which one element has a unique target shape, whereas one distractor element has a unique color, so that the task-irrelevant color singleton is equal or higher in saliency to the target (see, e.g., Theeuwes, 1992). At the same time, probes will measure the amount of attention at each element location. By using this dualtask procedure with postdisplay probes, this study is expected to determine how the task-irrelevant, bottom-up activation from the salient distractor is involved in visual search and how attention mechanisms select a predefined target, while ignoring that salient distractor. If subjects can still select only the target location in that situation, top-down control of attention in visual search must be quick and strong. On the other hand, any evidence of selection of a task-irrelevant singleton distractor location would reveal the importance of bottom-up factors on selection. Moreover, the amount of spatial attention at the locations of the target and the salient distractor might vary dynamically over time and/or practice. In this study, these possibilities will be examined.

\section{EXPERIMENT 1 Bottom-Up Influence on Spatial Selection}

The subjects were presented with a stimulus array containing a circle target among squares. The primary task

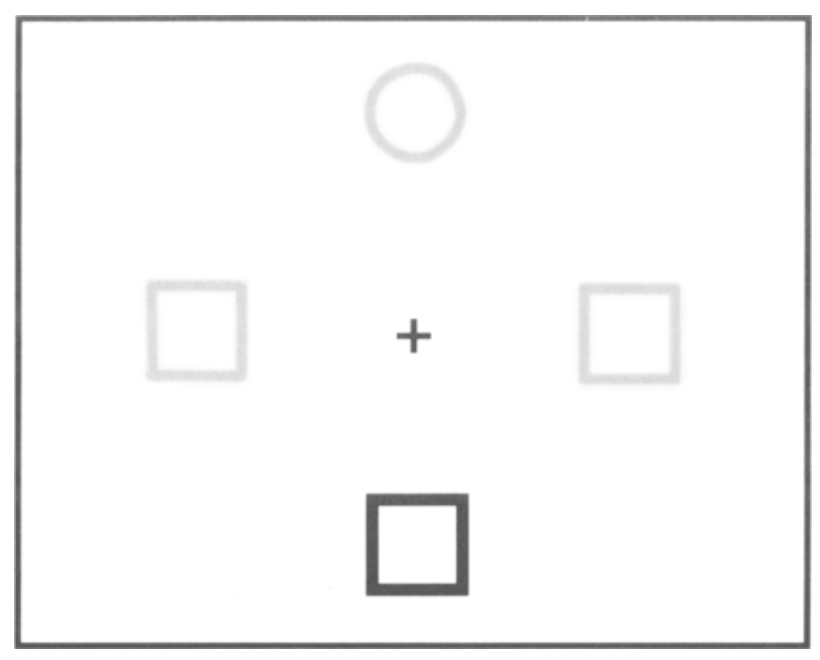

Figure 1. An example of the primary stimulus display in Experiment 1 . The circle was the target, and squares were distractors throughout all the trials. One distractor was colored differently from the others, and the color varied randomly for each trial. The different shades in the figure represent different colors, either red or green.

was to report whether the circle target was present or not. On the irrelevant feature dimension (color), all the stimuli were the same, except for one distractor (see Figure 1). The results of Theeuwes's $(1991,1992)$ experiments suggested that the task-irrelevant color singleton distractor should draw bottom-up attention more effectively than the shape singleton target. For each trial, the subjects did not know the target location or its color until the primary stimulus appeared, nor did they know the location of the singleton distractor. On half of the trials, the probe appeared with one of two different delays after the offset of the primary stimulus. The two different intervals between search display and probe onset (SOA) were chosen to be 60 and $150 \mathrm{msec}$, because preattentive processing was thought to occur within the first $100 \mathrm{msec}$ and attentive processing could occur after $100 \mathrm{msec}$ (see, e.g., Theeuwes, 1995b).

If a location with a salient singleton receives attention first, probe RTs at that location should be faster than those at the other locations in the short SOA condition, regardless of whether the unique feature is task-relevant or not. However, if a complete top-down guidance of selection is possible from the beginning of visual search, the location with the target feature should be more activated than the distractor location with a unique feature. On the other hand, if the distraction effects caused by a singleton distractor in many previous studies are not based on spatial selection (e.g., Folk \& Remington, 1998) or if the selection is made late, after all the elements are processed and identified, as is suggested in late-selection models (e.g., Allport, 1980; Deutsch \& Deutsch, 1963; Duncan, 1980; Norman, 1968; Posner, 1978; Shiffrin \& Schneider, 1977), there should be no differences in probe RTs at the different locations. 


\section{Method}

Subjects. Twenty undergraduates at Vanderbilt University participated in the experiment in partial fulfillment of a course requirement. All of them enjoyed normal or corrected-to-normal visual acuity and normal color vision.

Apparatus. The experiment was conducted on three AppleColor high-resolution RGB monitors controlled by Macintosh microcomputers. The screen resolution was $640 \times 480,69 \mathrm{dpi}$. The subjects responded via custom-built response keys that were connected to Strawberry Tree parallel interface cards. Responses were timed with clocks on the interface cards.

Stimuli. The primary search display in each trial consisted of four colored shapes, equally spaced around the fixation cross on an imaginary circle (see Figure 1). With a viewing distance of approximately $60 \mathrm{~cm}$, the radius of the imaginary circle spanned about $4.9^{\circ}$ of visual angle. The target was an outline circle, and the distractors were outline squares, each of which spanned about $2.2^{\circ}$ of visual angle. Each element was positioned on the horizontal or the vertical midline. In half the trials, the target was present, and in the other half, it was absent. However, a color singleton distractor was presented on every trial. In half the trials the color singleton was red, and the other three elements were green; in the other half, the singleton distractor was green, and the other elements were red. These trials were randomly intermixed throughout the whole experiment. The target and the singleton distractor appeared at each of the four locations equally often. The shades of red and green were

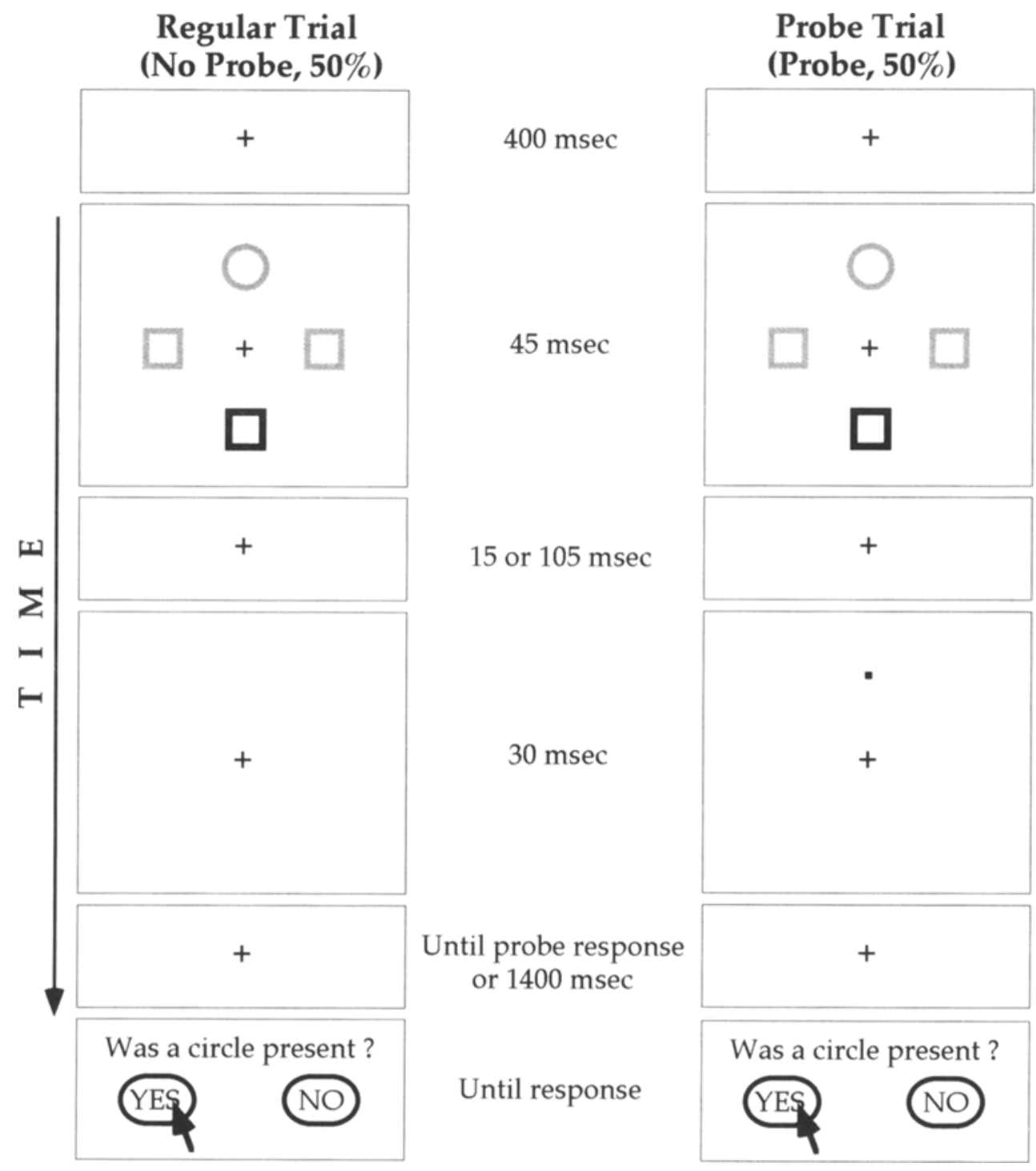

Figure 2. The sequence of displays in Experiment 1. The target was a circle. The different shades in the figure represent different colors, either red or green. At the beginning of each trial, a fixation cross appeared for $\mathbf{4 0 0} \mathrm{msec}$, and the primary display then appeared for $\mathbf{4 5} \mathrm{msec}$. In probe trials, a probe appeared after a brief interval of either 15 or 105 msec in the center of one of the four locations previously occupied by either the target or a distractor. After the subject responded to the probe or after a fixed interval had passed with no response, a question appeared, asking whether the target was presented. 
matched for luminance for each video monitor, using a Minolta Luminance ft- $\mathrm{I}$ deg light meter. Throughout the experiment, there was a black fixation cross at the center of the screen.

Procedure. The sequence of displays in a regular trial is illustrated on the left side of Figure 2. Half of the trials were of this type. Each trial began with the presentation of a fixation cross at the center of the screen. This was the subject's cue to fixate on the cross and prepare for the trial. After $400 \mathrm{msec}$, the four shapes (the primary stimuli) were presented. After $45 \mathrm{msec}$, the primary stimuli disappeared. On the regular trials, a blank screen with a fixation cross appeared for approximately $1,500 \mathrm{msec}$, followed by a display containing the question "Was a circle present?" 'Using a mouse, the subjects responded yes or no. They were instructed that speed was unimportant and that they should concentrate on accuracy. If the subjects responded incorrectly, they heard an error sound.

The remaining half of the trials were probe trials (the right side of Figure 2). The procedure was similar to that in the regular trials, except that a small black dot (the probe, approximately $0.2^{\circ}$ of visual angle) appeared very soon after the primary stimulus. The probe appeared at the center of one of the four locations formerly occupied by a search element and remained visible for $30 \mathrm{msec}$. The probe appeared at each of the four locations equally often, regardless of the target's location. In response to the probe, the subjects pressed a button as quickly as possible. The delay between primary stimulus onset and probe onset (SOA) was selected randomly for each trial to be either 60 or $150 \mathrm{msec}$. After the probe offset, the display was blank for $1,400 \mathrm{msec}$. If the subjects missed the probe and did not press the button during the 1,400-msec interval or if they pressed it when no probe appeared, they heard a different error sound.

Each subject worked through at least 25 practice trials before data collection. More practice was given if the subject or the experimenter judged it necessary. The subjects were given a break every 40 trials. Each subject received a total of 768 trials beyond practice. The mouse was used by the dominant hand for responding in the primary task (identifying the target letter). Response to the probe (pressing a button) was done by the nondominant hand.

\section{Results}

Correct response rates were above $98 \%$ in the primary task and above $97 \%$ in the probe task. Since the error rates in the primary and the probe tasks were very low, they were not analyzed further.

Probe RTs for each subject were sorted into cells according to the conditions in each trial. RTs more than 3.5 standard deviations ( $S D$ s) from the mean for that cell were trimmed iteratively. They were fewer than $2 \%$ of all the observations. The mean RTs from trials with both a correct primary response and a correct probe response

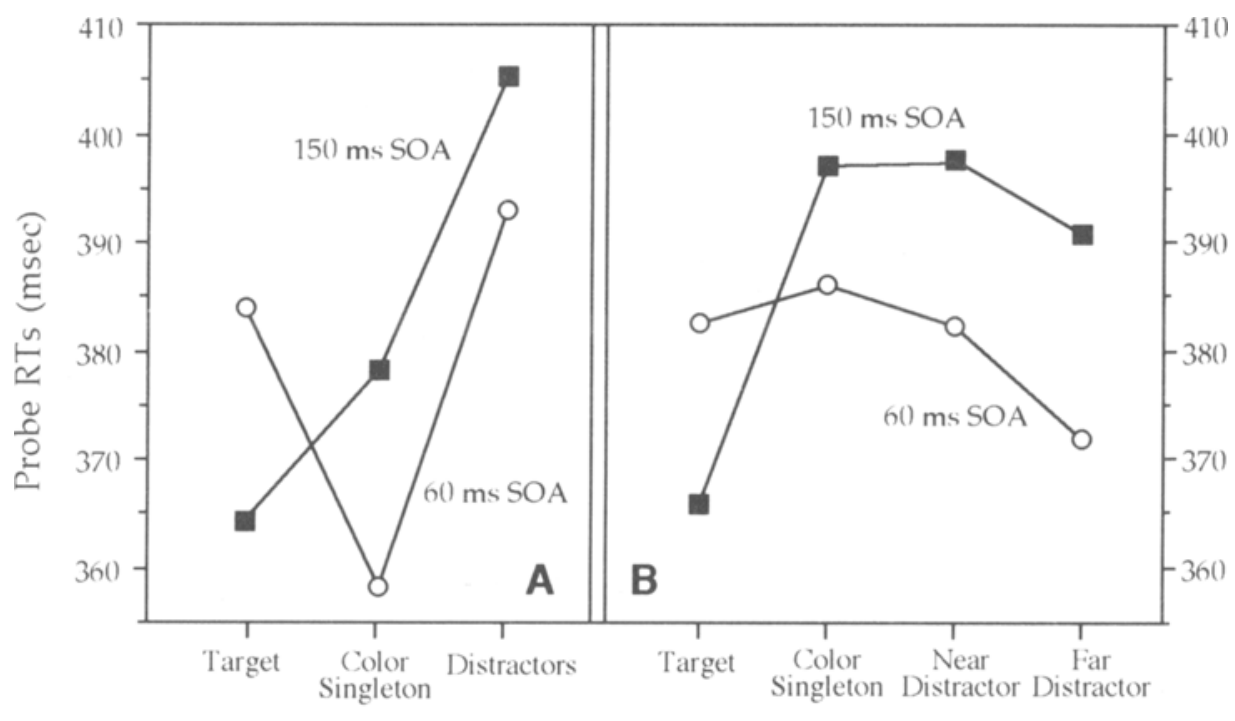

Probe Location
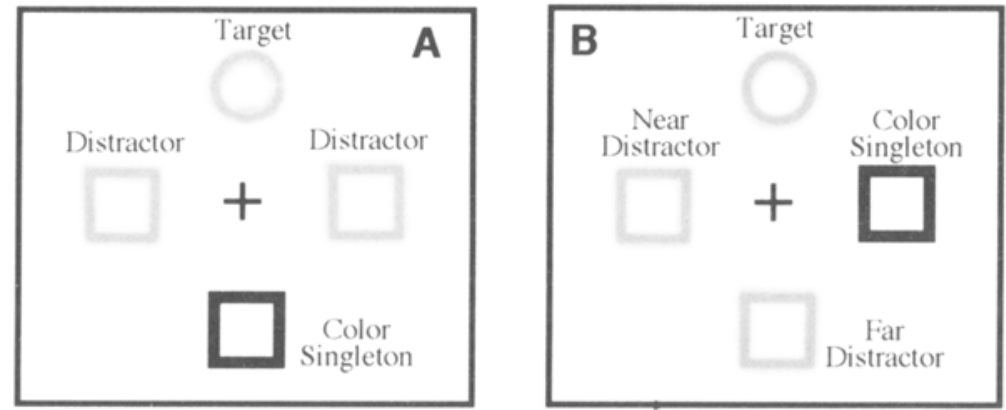

Figure 3. Mean response times to probes that appeared at the location occupied by the target, a color singleton distractor, or target-color distractors, with two SOAs. The left panels (A) show data from trials in which the target and the color singleton distractor were located far from each other, whereas the right panels (B) show results from trials in which they were positioned next to each other. 
were subjected to an analysis of variance (ANOVA). The primary stimulus array could be displayed in two different types of configuration - one in which the target and the color singleton appeared on opposite sides of the display, and one in which they were located next to each other (see the bottom panels of Figure 3). Thus, separate ANOVAs were performed for each configuration.

The upper left panel in Figure 3 shows the mean RTs to probes at each element location, when the target and the color singleton distractor were located far from each other. The first ANOVA included three levels of probe location (target, color singleton distractor, or target-color distractor) and two levels of SOA (60 or $150 \mathrm{msec})$ as factors. The analysis showed a significant main effect of probe location $[F(2,38)=8.48, p<.001]$; the subjects took longer to respond to the probe at the target-colored distractor locations than at the target or color singleton distractor locations. There was no main effect of SOA $(F<.5)$, nor did the interaction between SOA and probe location achieve significance $[F(2,38)=2.15, p=.13]$. Separate analyses for each SOA condition were performed. There was a significant main effect of probe location both for the 60 -msec SOA $[F(2,38)=3.29, p<$ $.05]$ and for the $150-\mathrm{msec} \operatorname{SOA}[F(2,38)=6.45, p<.01]$. A planned comparison showed that, with the $60-\mathrm{msec}$ SOA, RTs for the color singleton distractor location were significantly faster than those for the other distractor locations $[F(1,19)=8.63, p<.01]$. However, there was no significant difference between the color singleton and the target locations $[F(1,19)=3.08, p=.1]$. With the $150 \mathrm{msec}$ SOA, probe RTs at the target-color distractor locations were significantly longer than those at the target location $[F(1,19)=16.78, p<.001]$ and at the singleton color distractor location $[F(1,19)=6.44, p<.05]$.
For the other configuration of search display (upper right panel in Figure 3), another ANOVA was performed, with four levels of probe location (target, color singleton, distractor next to the target, or distractor next to the singleton distractor) and two levels of SOA (60 or $150 \mathrm{msec}$ ) as factors. The main effects did not achieve significance, either for probe location $[F(3,57)=2.09$, $p=.11]$ or for $\operatorname{SOA}[F(1,19)=1.23, p=.28]$. There was no significant interaction $[F(3,57)=2.14, p=.11]$. Separate analyses for each SOA condition were performed. With the $60-\mathrm{msec} \mathrm{SOA}$, there was no main effect of probe location $(F<1)$. With the 150 -msec SOA, however, there was a significant main effect of probe location $[F(3,57)=3.95, p<.05]$. A planned comparison showed that the subjects responded to the probe more quickly at the target location than at the other locations $[F(1,57)=$ $11.34, p<.005]$.

A separate analysis was performed on the mean RTs for the three probe locations (color singleton, targetcolor distractor positioned next to the color singleton, and target-color distractor positioned far from the color singleton) and for the two SOAs (60 and $150 \mathrm{msec})$ in the target-absent condition (see Figure 4). The main effects did not achieve significance, either for probe location $(F<1)$ or for SOA $[F(1,19)=2.76, p=.11]$. There was no significant interaction $[F(2,38)=2.22, p=.12]$. Contrary to the results from the target-present condition, probe responses were not faster at the color singleton distractor location, even with the early SOA $(60 \mathrm{msec})$.

\section{Discussion}

In the early stage of visual processing (60-msec SOA), the subjects could not allocate attention to the circle target location. Instead, their attention went to the singleton

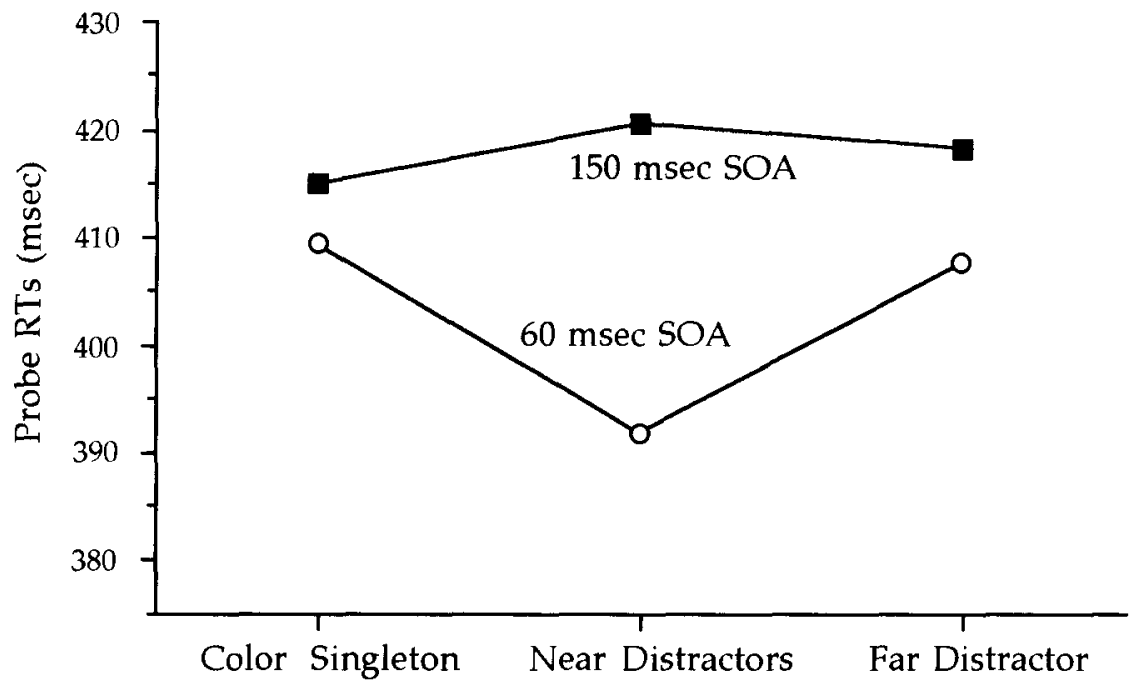

Probe Location

Figure 4. Mean probe response times, organized by distance of the probe from the color singleton, when the target was absent. 
distractor location, as long as it was not adjacent to the target. Note that the subjects in Kim and Cave's (1995) simple feature search task showed faster probe RTs at the circle target location than at any of six square distractor locations with a 30-, 60-, or 90-msec SOA when there was no color singleton distractor. Thus, the absence of a benefit in responding to the probe at the target location at the short SOA in the present experiment suggests that the existence of the color singleton distractor prevented the subjects from allocating their attention to the target location early in visual processing.

Soon thereafter (150-msec SOA), the location of the target more activated than the color singleton or any of the other distractors, which implies that the top-down component of attentional control can override the interference from the color singleton distractor. In this sense, these results appear to be in line with Theeuwes's (1995b) finding, in which abrupt onsets interfered with searching for a simple target before a 100 -msec SOA, but not after a 100-msec SOA.

As is shown in Figure 3, with a 60-msec SOA, the color singleton drew attention to its location only when it was located relatively far away from the target. This pattern is the opposite of what might be predicted from Theeuwes's (1995b) claim that attention gradually zooms in on the target location, which would imply more attention to distractors near the target. Nonetheless, the pattern of attention in this experiment might be explained by assuming that the operations of the preattentive, parallel stage are local. That is, the comparisons and competitions to detect singletons might occur within a limited area. (See Cave, in press, and Cave, Kim, Bichot, \& Sobel, 1999, for a neural network model of visual selection that relies on a parallel-distributed selection mechanism based on local operations, as well as for earlier models by Koch \& Ullman, 1985, and Duncan \& Humphreys, 1989.) The bottom-up attentional mechanism might only allocate attention to a location if its features differ from the features at nearby locations and if those surrounding features are all uniform. Thus, when the color singleton is close to the target, neither of them is locally salient enough to draw attention. On the other hand, when they are far from each other and, thus, locally surrounded by elements that are similar to one another, attention can be allocated to a more salient element - the color singleton first and then the target (see, e.g., Theeuwes, 1992). Note that Theeuwes's (1995b) singleton distractors were abrupt onsets, which probably draw attention so readily that they are less affected by these local interactions.

This interpretation appears to be consistent, not only with the results of Experiment 1, but also with Theeuwes's (1995a, 1995b) results, in which a singleton distractor produced longer RTs (more interference) when it was positioned near the target. When the singleton distractor is located near the target, both the target and the singleton distractor would produce less bottom-up activation, and thus, spatial attention to the target location should be weaker or develop more slowly than when the singleton distractor is located farther away from the target.

The lack of attention to a singleton distractor near the target might also be attributed to top-down inhibition of distractor locations. Cave and Zimmerman (1997) found that distractors near a visual search target were inhibited more strongly than those farther from the target, although that inhibition seemed to be linked to extended practice of the search task. Caputo and Guerra (1998) reported a similar finding. Even though the top-down mechanism is not able to select the target location at the early SOA, it might be able to inhibit the nearby distractor locations enough to prevent them from being strongly activated.

Overall, subjects seem to find it necessary to select the target when it is present, and they apparently cannot select the target without first selecting the singleton distractor. There is, however, an alternative account that must be considered. Subjects might choose a response just by counting the number of singletons. If only one is present, it is the color distractor, and a no response is correct. If there are two singletons, one is the target, and a yes response is correct. If there were no evidence of spatial selection in this experiment, the possibility of this alternative strategy would be a cause for concern. However, the differences in probe RTs in this experiment show that the subjects are selecting the target location, indicating that they are not relying on a preattentive singleton-counting strategy. A singleton-counting strategy would have been even easier in Kim and Cave's (1995) feature search, which included search tasks, stimuli, and probe tasks similar to those in Experiment 1. In that experiment, target-present trials had one singleton, and target-absent trials had zero. Yet, when the subjects searched for a circle target among square distractors in that experiment, probe RTs showed that they selected the target location in the course of the visual search.

Nevertheless, the action of spatial attention in Experiment 1 and in Kim and Cave's (1995) feature search is not necessarily relevant to the response, as it is in experiments such as Theeuwes's $(1991,1992)$. It may be that subjects base their responses solely on the number of singletons they count, whereas the probe RTs reflect a bottom-up allocation of attention that is beyond their control or an automatic selection of the target after a response is generated. This conclusion, however, is inconsistent with the general pattern of results from Kim and Cave (1995), which demonstrated that spatial selection occurs in some feature searches, but not in others. The fact that subjects performed some searches without spatial selection shows that subjects employ spatial attention only when they find it useful for the primary task. Thus, we know that the subjects in Experiment 1 are relying on spatial selection in detecting and identifying the shape target. Although Experiment 2 was motivated by other concerns, it will also supply additional evidence that the allocation of attention in this task is not automatic and unavoidable and that the evidence for spatial selec- 
tion therefore indicates that selection plays an important role in these searches. Thus, even though there may be a strategy that would allow subjects to perform this task without attention, the evidence shows that they are not using it. Subjects are unable to detect the targets without attention by counting singletons, they find the tasks easier with attention, or they do not realize that the task could be done without attention.

The data from the target-absent trials are also somewhat surprising. If selection occurs in a purely bottom-up fashion at the preattentive level, as Theeuwes claimed, there should be a strong attentional capture by the color singleton in the short SOA, which was not the case in the target-absent condition. In Theeuwes's (1992) experiment, the singleton target was always presented; there was no target-absent trial in his experiments. One block always contained only the target singleton (no-distractor condition), whereas the other block displayed both the target and the singleton distractor. In contrast, the subjects of the present experiment did not know beforehand whether there would be one or two singletons in the display. Although the subjects did not use information about the number of singletons to detect the target without attention, they may have used it to determine when not to search. Once they discovered that only one singleton (distractor) was present on a trial, they might have exerted top-down control to block the bottom-up activation of distractor locations. Of course, if subjects can determine that search is unnecessary by counting singletons, they should also be able to choose the correct response on the basis of only the singleton count. Perhaps the count is inaccurate enough that they will only decide that a target is present after selecting and identifying it.

As is shown in Figure 4, probe RTs in the target-absent condition were somewhat slower than those in the targetpresent condition. These probe responses might be slower because subjects are inhibiting all the distractor locations when only one singleton appeared. Another possibility is that a negative target response (no target) in the primary task interferes with a positive probe response (yes probe) and delays the probe response. A third possibility is that subjects might engage in extra search effort (e.g., rechecking items) when they do not find a target. Any of these effects would slow all the probe responses in the targetabsent condition, regardless of probe location.

In the present experiment, there was a trend toward a significant interaction between probe location and SOA for both configurations, although it did not reach significance in either case. Thus, it is not clear whether the allocation of attention varies between these two SOAs. Experiment 2 will provide additional data to resolve this issue. It also is conceivable that complete top-down control might be possible after extended practice. The 25 practice trials and 768 main trials that each subject received in the present experiment might not be enough to develop a strong top-down selectivity. The subjects might either inhibit the task-irrelevant bottom-up activation and/or increase top-down selectivity toward the to-beknown target feature more effectively or more quickly after practice. The next experiment will address this issue, using the same task as that in the present experiment, but with multiple sessions for extended practice.

\section{EXPERIMENT 2 The Role of Practice in Visual Selection}

Experiment 1 showed that attention is directed to the color singleton distractor location in one type of display configuration. Also, even in the other type of configuration, the lack of attention at the target location with the short SOA suggests that the color singleton interferes with selecting the target location. However, this bottomup interference might be diminished by practice with a consistent mapping between stimuli and responses.

As was mentioned earlier, Theeuwes (1992) found that the presence of a task-irrelevant color singleton distractor interfered with simple feature search for a circle among squares. Moreover, he showed that the interference effect persisted even after extensive practice. In his study, 8 subjects searched for a green circle among square distractors. In one half of the blocks, all the squares were green. In the other half of the blocks, one of the squares was red. The subjects received 1,728 experimental trials after 288 practice trials. The experimental trials were divided into three sessions. He found no differences across sessions and concluded that top-down selectivity is impossible at the parallel preattentive stage (i.e., before $100 \mathrm{msec}$ ), even after extensive practice.

As was mentioned earlier, however, the longer RTs in the color singleton distractor condition than in the no-singleton distractor condition do not necessarily indicate a complete lack of top-down selectivity. Adding the salient color singleton distractor could cause a decrease of the bottom-up activations at the target location and an increase of the bottom-up activations at the singleton distractor location. Thus, top-down activation may be working to block the bottom-up effects of the singleton distractor but may be unable to completely prevent it from interfering.

In the present experiment, we will further investigate whether and how top-down control changes with consistent and extended practice. Extensive practice might enable focal attention to be guided to the target location more efficiently in the preattentive stage. If so, probe RTs at the target location should become relatively faster than at the other locations with more practice, even in the short SOA condition. Also, practice might allow bottom-up activations from the color singleton distractor to be blocked more effectively in the preattentive stage. If so, the probe RTs at the singleton distractor location should equal probe RTs at other distractor locations.

The predictions above assume that spatial attention is required to prevent distractors from interfering with target processing, even after extensive practice. On the other hand, practice may produce an ability to process and iden- 
tify stimuli without attention. If this is true, spatial attention should not be directed to the target location either.

In the present experiment, each subject performed the same tasks as those used in Experiment 1. Instead of participating in one session, however, they were in six sessions, one session per day.

\section{Method}

Subjects. Ten subjects ( 7 men and 3 women), ranging in age from 21 to 54 years, participated as paid volunteers. All had normal or corrected-to-normal visual acuity and normal color vision.

Apparatus, Stimuli, and Procedure. The apparatus, visual stimuli, and task were identical to those in Experiment 1 . The only difference between the two was that the subjects in this experiment were run in multiple sessions, one session per day. All the subjects were tested for six experimental sessions. Before data collection at the first experimental session, each subject worked through more than 300 practice trials. Each experimental session consisted of 768 trials and took approximately $40 \mathrm{~min}$. All the subjects finished all the sessions within 10 days.

\section{Results}

Correct response rates were above $98 \%$ in the primary task and above $98 \%$ in the probe task. RTs more than $3.5 \mathrm{SD}$ s from the mean were trimmed iteratively. They were fewer than $2 \%$ of all the observations.

The mean RTs from trials with both a correct primary response and a correct probe response were subjected to ANOVAs. As in Experiment 1, the two different configuration conditions were analyzed separately, with probe location and session as factors.

Figure 5 shows the mean RTs to probes at each element location when the target and the color singleton distrac-

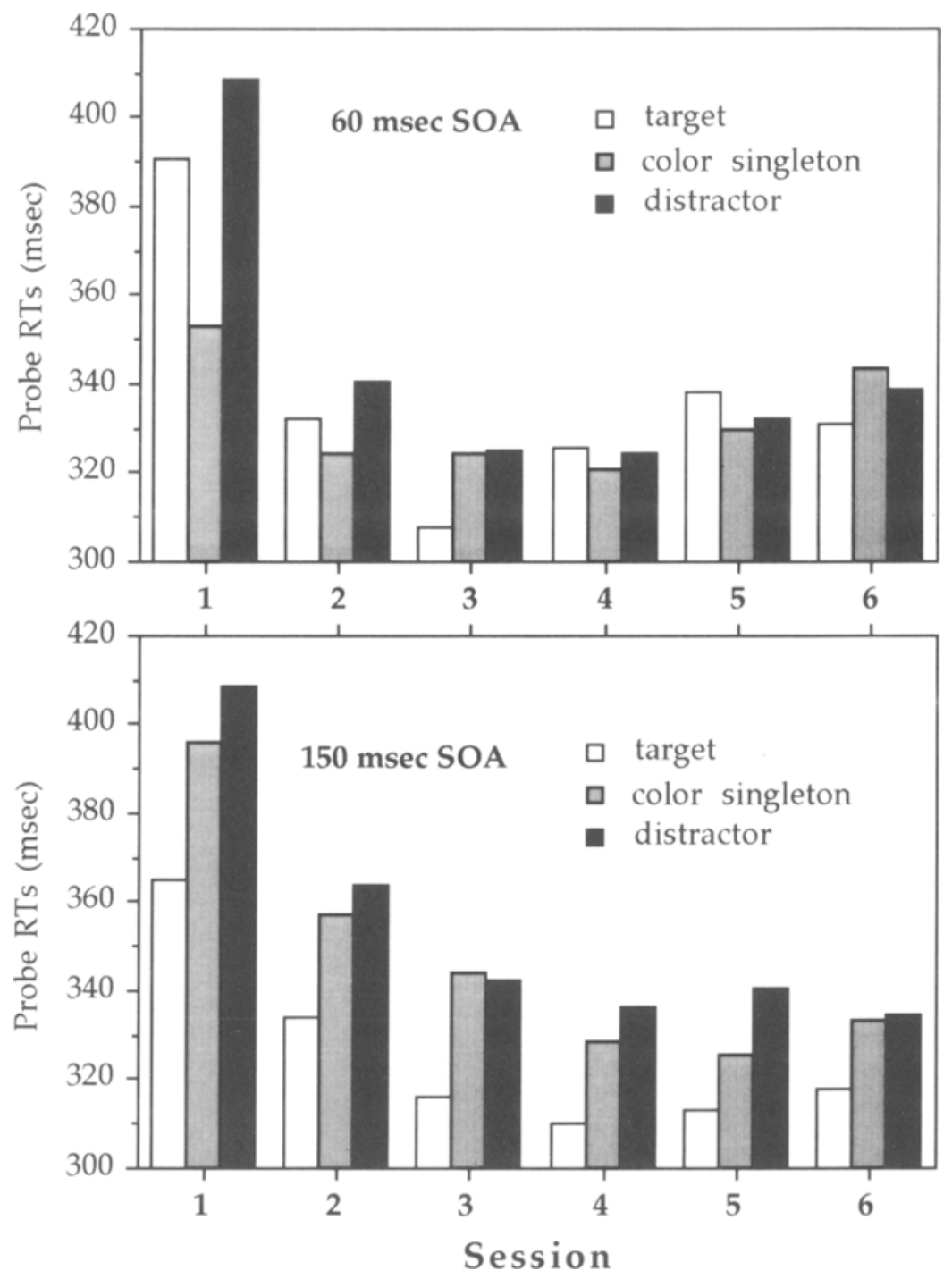

Figure 5. Mean response times to probes that appeared at the location occupied by the target, a color singleton distractor, or target-color distractors, when the target and the color singleton distractor were positioned far from each other. 
tor were positioned far from each other. The first ANOVA included only data from the first session, to confirm the results of the previous experiment (see Session 1 in Figure 5). It included three levels of probe location (target, color singleton distractor, or target-color distractor) and two levels of SOA (60 or $150 \mathrm{msec}$ ) as factors. The analysis showed a significant main effect of probe location $[F(2,18)=5.70, p<.05]$ and a significant interaction between SOA and probe location $[F(2,18)=4.62, p<$ $.05]$. The significant interaction confirms the conclusions, from Experiment 1, that the color singleton received more attention at the early SOA and the target received more attention at the later SOA.

For each SOA, the data from all six sessions were analyzed, with probe location and session as factors. In the 60-msec SOA condition (the top panel of Figure 5), there was a significant main effect of session $[F(5,45)=3.27$, $p<.05]$; the subjects took longer to respond to the probe in the first session than in the other sessions. There was no main effect of probe location. More important, however, there was a significant interaction between session and probe location $[F(10,90)=2.29, p<.05]$, suggesting that the relative attentional strength at each location changed with practice. As is shown in the top panel of Figure 5, the subjects showed faster probe RTs at the color singleton distractor location than at any other locations in the first session. In the later sessions, however, probe RTs at the color singleton were not generally much faster than those at the other locations. Individual ANOVAs for each of the six sessions showed that there was a significant probe location effect only in Session $1(p<.05)$, and not in the other sessions.

In the 150-msec SOA condition (the bottom panel of Figure 5), there was a highly significant main effect on probe RT for both probe location $[F(3,27)=17.14, p<$ $.001]$ and session $[F(5,45)=6.86, p<.001]$. There was no interaction between the two: Probe RTs were faster for the target location than for other locations throughout the sessions. The data were also analyzed separately for each of the six sessions. In all the sessions, the subjects generally showed faster probe RTs at the target location than at the other locations, although only the first four sessions reached significance ( $p<.05$ for the first four sessions; $p<.1$ for the last two sessions).

Separate ANOVAs were performed for the other configuration of search display in which the target and the color singleton distractor were located next to each other. Once again, an ANOVA with only the first session was performed (see Session 1 in Figure 6). The analysis showed no significant main effect for probe location $[F(3,27)=$ $1.6, p=.21]$ or for SOA $(\mathrm{F}<1)$. However, there was a marginally significant interaction between SOA and probe location $[F(3,27)=2.53, p=.08]$. In general, the results were once again similar to those from Experiment 1.

With all six sessions included, the data were analyzed separately for each SOA condition. In the 60-msec SOA condition (see the top panel of Figure 6), the subjects re- sponded to the probe more quickly after the first session $[F(5,45)=4.14, p<.01]$. With the data from all six sessions included, the ANOVA showed a main effect of probe location $[F(3,27)=3.48, p<.05]$, which was not found in Experiment 1. Separate post hoc pairwise comparisons showed faster probe RTs at the target and the color singleton locations than at the far distractor location. However, these effects were not significant when the data were analyzed separately for each session. There was no significant main effect of probe location in any of the sessions, except Session $4[F(3,27)=3.38, p<$ $.05]$, in which the subjects showed faster probe RTs at the target and the color singleton distractor locations than at near (not far) distractor locations. Thus, the significant main effect of probe location with the data from all six sessions may not represent a pattern that holds across all six sessions. The interaction between session and probe location was not significant.

In the 150-msec SOA condition (the bottom panel of Figure 6), there were highly significant main effects on probe RT from both probe location $[F(3,27)=23.01, p<$ $.001]$ and session $[F(5,45)=9.45, p<.001]$. There was no interaction between the two. Contrary to the short SOA condition, in this case, the main effect of probe location was due only to faster probe RTs at the target location. Also, the lack of an interaction indicated that consistent practice did not change the relative attentional strength at each location. The data were also analyzed separately for each session. In all six sessions, the subjects showed significantly faster probe RTs at the target location than at the other locations.

Separate ANOVAs were performed for the target-absent condition. Once again, an ANOVA with only data from the first session was performed, to confirm the results of the previous experiment (see Session 1 in Figure 7). The analysis showed no significant main effect for probe location $[F(2,18)=1.1, p=.37]$ or for SOA $(F<1)$. There was no significant interaction $(F<1)$. These results were the same as those from Experiment 1, in that probe responses at the color singleton distractor location were not faster than those at the target-colored distractor locations when the target was absent.

With all six sessions included, the data from the targetabsent trials were analyzed separately for each SOA condition. In the 60-msec SOA condition (see the top panel of Figure 7), the analysis showed that probe RTs were slower in the first session than in the other sessions $[F(5,45)=4.66, p<.01]$. There was neither a significant main effect for probe location $(F<1)$ nor a significant interaction between probe location and session $[F(10,90)=$ $1.74, p>.08]$. With the $150-\mathrm{msec}$ SOA (bottom panel of Figure 7 ), the subjects once again responded more slowly to probes in the first session than in the other sessions $[F(5,45)=7.12, p<.001]$. Even though there was a significant main effect of probe location $[F(2,18)=$ $4.49, p<.05]$, the overall difference in mean RTs at each location was very small (344 $\mathrm{msec}$ at singleton distrac- 

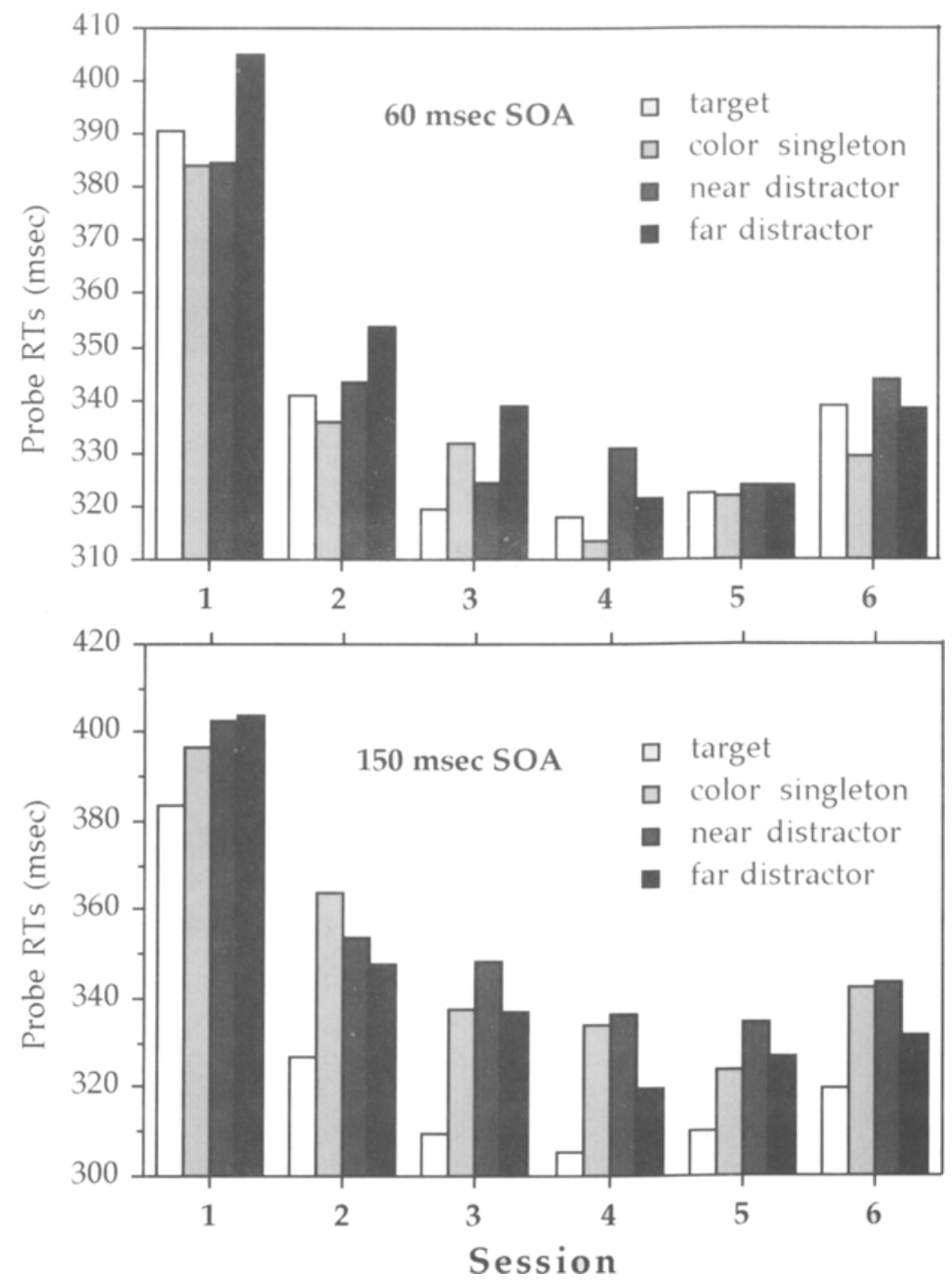

Figure 6. Mean response times to probes that appeared at the location occupied by the target, a color singleton distractor, or target-color distractors, when the target and the color singleton distractor were located next to each other.

tor, $349 \mathrm{msec}$ at near distractor, and $350 \mathrm{msec}$ at far distractor). Separate ANOVAs for each session showed no significant main effect for probe location. The interaction between session and probe location was not significant $(F<1)$.

\section{Discussion}

First of all, the first sessions of the present experiment confirm the somewhat surprising finding from the previous experiment that the singleton distractor captured attention at the short SOA when it was far from the target, but not when it was next to it. In the long SOA, the target location received more attention than did the other locations, as in the previous experiment. Also, the first session replicates the target-absent data of Experiment 1; the color singleton did not draw attention to its location.
The present experiment was designed to understand whether and how extended practice influences spatial attention in a visual search task, especially when a salient, task-irrelevant distractor is presented along with a target. First, the results from the $150-\mathrm{msec}$ SOA condition in both configurations show that spatial attention selects the target location over distractor locations, even after extended practice. These results are in line with Cave and Zimmerman's (1997) finding that selection of the target location over distractor locations was not diminished, even after large amounts of practice (more than 80,000 trials).

Although probe RTs in the $150-\mathrm{msec}$ SOA condition showed that practice does not alter allocation of spatial selection of the target location, the results from the 60 msec SOA condition suggest that changes did take place in spatial attention with practice. As is shown in the top 

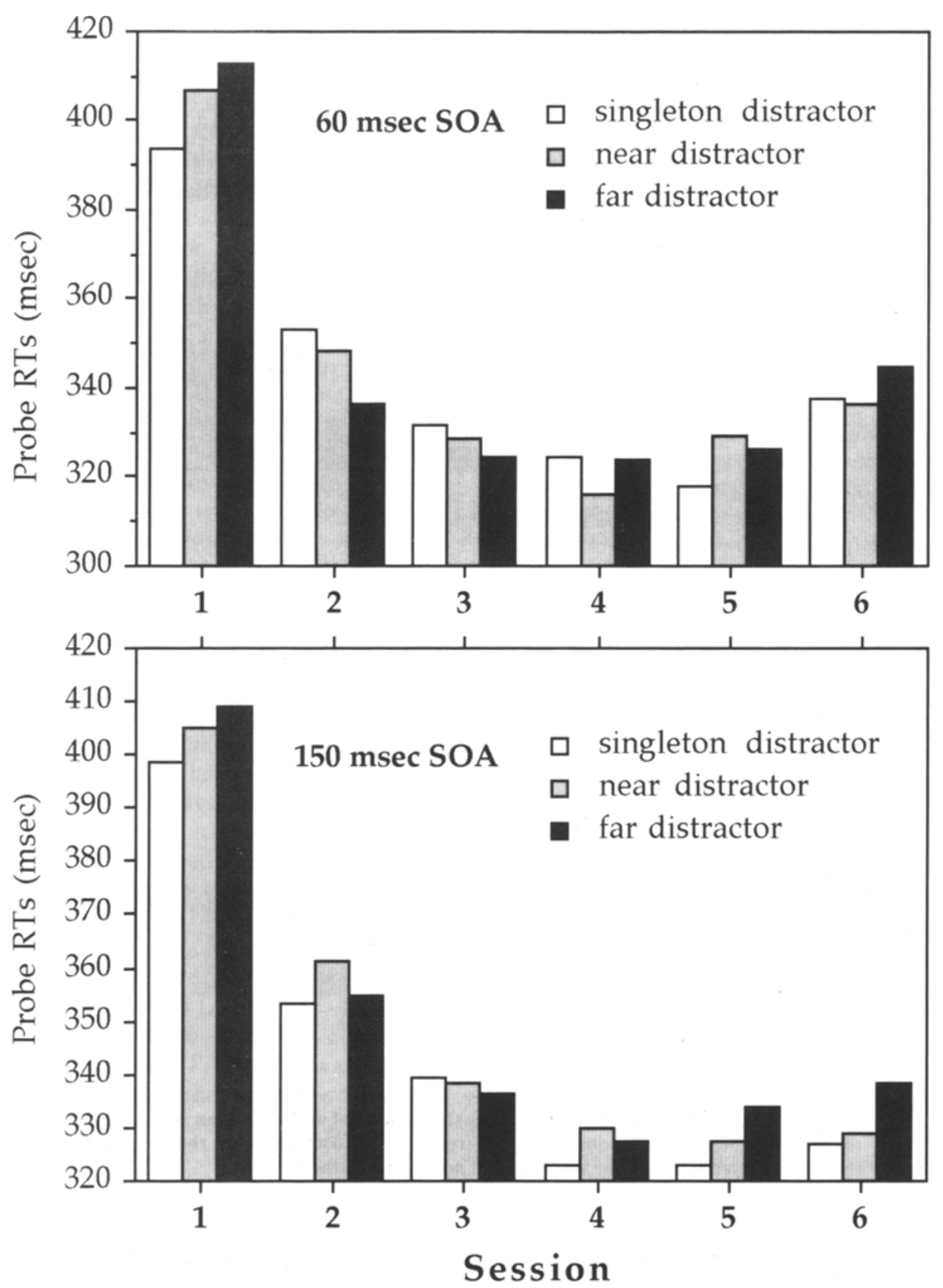

Figure 7. Mean response times to probes that appeared at the location occupied by a color singleton distractor or target-color distractors with two SOAs, 60 msec (top) and 150 msec (bottom), when the target was not presented.

panel of Figure 5, the color singleton distractor positioned far from the target drew more attention in the first session, but not in the later sessions. That is, after the subjects received a full session of practice, they were apparently able to ignore the salient, task-irrelevant color singleton distractor more efficiently or more quickly.

Also, note that, with the 60-msec SOA, the color singleton did not capture attention during any session in the target-absent condition (see the top panel of Figure 7). In the target-absent condition, the color singleton distractor is the only singleton in the display. The color singleton is the only item that has high bottom-up activation and, thus, should capture attention. Nevertheless, the data showed no indication of attentional capture by the color singleton at the $60-\mathrm{msec}$ SOA. These results suggest that attention is not driven only by bottom-up factors at the preattentive stage of processing.

The present experiment used only two SOAs to measure spatial attention at the different stages of visual processing. If attentional capture occurs in a bottom-up 
fashion earlier than 60-msec SOA with more practice, the present experiment could miss it. Nevertheless, consistent practice is producing more efficient selection in one way or another. The extra practice might allow subjects to block out the task-irrelevant bottom-up activation of the singleton distractor, increase the activation of the target features in a top-down fashion, or both.

Interestingly, even though the present data suggested that attentional capture by a salient singleton distractor at the preattentive level (i.e., before the 100-msec SOA) can be prevented by extended practice, they also showed that attention is not directed to the target location more quickly at the $60-\mathrm{msec}$ SOA with practice in either configuration, which is consistent with Theeuwes's (1992) data. Even after extended practice, the target location was not selected at the short-SOA condition. Presumably, search efficiency can be measured by search accuracy as well as search speed. Although it is not clear why the extra practice did not produce any benefit for target location over distractor locations at the short SOA, it might increase search efficiency-accuracy-by limiting the interference from the color singleton distractor in early visual processing.

The fact that the color distractor is not selected after extended practice underscores the point raised after Experiment 1 , that attention to the color singleton is not automatic and unavoidable. However, a new possibility arises with Experiment 2: Perhaps the practice allows subjects to learn to detect the target by counting singletons preattentively. In other words, perhaps attention is no longer allocated to the color distractor location because subjects have learned to eliminate the need for attention by counting singletons. Under this account, however, it is difficult to explain why subjects still select the target location even after practice. One possibility, mentioned earlier, is that the target location might always be selected, even when selection is unnecessary for the task, but the feature search results from Kim and Cave (1995) argue against that explanation. Besides, even if subjects are learning to determine the presence of the target by counting singletons, it is difficult to see how this knowledge of the target's presence should allow practiced subjects to select the target without first selecting the singleton distractor, given that they were unable to select the target first before they learned to count singletons.

In summary, it is possible, in theory, to perform these tasks without attention, if subjects can detect and count singletons preattentively. However, the probe RTs show that subjects are performing the task in the manner suggested by the instructions: They are searching for the location with the target feature and selecting it.

\section{GENERAL DISCUSSION}

The present experiments were designed to examine the nature of distractor interference in visual search and the possibility of top-down selectivity early in visual processing. Using spatial probes, these experiments mea- sured spatial attention at the location of each search element, including the target and a singleton distractor. The probe RTs suggest that the interference from the singleton distractor is the result of spatial selection of its location. The color singleton distractor captured attention to its location soon after the stimulus appeared, but only when it was located far from the target. This bottom-up activation at the color singleton distractor location was overridden by top-down attention some time later, indicating temporal changes in the influence of the two different attentional components. This tendency to select the target location over distractor locations occurred even after extended practice.

Our previous study (Kim \& Cave, 1995), without a color singleton, showed that the target location was selected even at very short SOAs, such as 30 or $60 \mathrm{msec}$. With a color singleton distractor, however, evidence of selection of the target location arises only after a $150-\mathrm{msec}$ delay, and not after a 60-msec delay. Moreover, even though the interference from the singleton distractor seems to be blocked more efficiently with practice, there is no indication that more practice speeds up target selection. Whether or not the color singleton's location is actually selected, its presence seems to delay selection of the target even after extended practice, as would be expected, given Theeuwes's (1992) results.

Although Theeuwes's (1992) experiments indicate that a singleton distractor will draw attention automatically, other search experiments have shown no automatic selection of singletons (Folk \& Annett, 1994; Jonides \& Yantis, 1988; Yantis, 1995). Bacon and Egeth (1994) tried to explain the apparent discrepancy between the two positions by suggesting that, when subjects search for a salient singleton target, they can go into singleton detection mode, in which they attend to the singleton with the highest saliency, regardless of feature dimension. Since the subjects in our experiments searched for a shape singleton target, they might be in singleton detection mode, and thus, the color singleton could receive attention by a feature contrast detector. Although the present data are generally compatible with the singleton mode account, they require an additional assumption, that a singleton distractor draws attention less effectively when it is near a singleton target.

These results shed new light on the questions about distractor interference raised by Folk and Remington (1998). After a series of spatial cuing experiments (see, also, Folk \& Annett, 1994; Folk \& Remington, 1996; Folk, Remington, \& Johnston, 1992; Folk, Remington, \& Wright, 1994), they concluded that singleton distractors were not automatically capturing spatial attention but were adding to the filtering costs associated with selecting a target among distractors, without receiving attention themselves. The present experiments demonstrate that, at least under some conditions, attention is allocated to a singleton distractor. In most of Folk et al.'s experiments, the delay between the singleton distractor and the target was $150 \mathrm{msec}$. If the dynamics of spatial se- 
lection is similar in the two tasks, the present results suggest that attention would already have been deallocated from the singleton by that time. Even when Folk and Remington (1998) used a delay of $50 \mathrm{msec}$ (Experiment 4), however, they found no evidence of selection of the distractor location. There may be some aspect of Folk and Remington's (1998) cuing task that allows subjects to block attention to a singleton distractor, or the test in their Experiment 4 may not have been sensitive enough to detect the effects of spatial attention to the distractor. Whatever the explanation for the differences, our results are still consistent with Folk et al.'s conclusion that attentional capture can be affected by top-down factors.

Overall, our present findings show that, even at the preattentive, parallel stage and with a search array that covers a large portion of the visual field, visual selection depends, in large part, on interactions within limited regions. The selection of a singleton distractor can be prevented by a nearby target, but not by a target that is farther away. These data also suggest that top-down selectivity is not completely impossible at the preattentive stage, despite the claims of Theeuwes $(1991,1992,1995 b)$. The absence of early selection of the color singleton in the targetabsent condition suggests that top-down components of attention somehow exert control, perhaps through blocking bottom-up activations of the color singleton. The fact that practice also prevents the selection of the color singleton provides further evidence for some degree of topdown control at the early stage of visual processing. If the practice effects do reflect some top-down influence, however, that influence is not strong enough to cause the target to be selected early in the search. Further experiments measuring how the allocation of spatial attention develops over time will be necessary, to work out the interaction between bottom-up and top-down mechanisms in visual search.

\section{REFERENCES}

AllPORT, D. A. (1980). Attention and performance. In G. Claxton (Ed.), Cognitive psychology: New directions (pp. 112-153). London: Routledge \& Kegan Paul.

BACON, W. F., \& EGETH, H. E. (1994). Overriding stimulus-driven attentional capture. Perception \& Psychophysics, 55, 485-496.

Bichot, N. P., CAVE, K. R.. \& PAshler. H. (1999). Visual selection mediated by location: Feature-based selection of noncontiguous locations. Perception \& Psychophysics, 61, 403-423.

Bundesen, C. (1990). A theory of visual attention. Psychological Review, 97, 523-547.

Caputo, G., \& Guerra, S. (1998). Attentional selection by distractor suppression. Vision Research, 38, 669-689.

CAVE, K. R. (in press). The FeatureGate model of visual selection. Psychological Research.

Cave, K. R., Kim, M.-S., Bichor, N. P.. \& Sobel, K. V. (1999). Visual selection within a hierarchical network: The FeatureGate model. Manuscript submitted for publication.

CAVE, K. R., \& WolfE, J. M. (1990). Modeling the role of parallel processing in visual search. Cognitive Psychology, 22, 225-271.

Cave. K. R.. \& Zimmerman. J. M. (1997). Flexibility in spatial attention before and after practice. Psychological Science, 8, 399-403.

Cepeda, N. J.. Cave, K. R., Bichot, N. P., \& Kim, M.-S. (1998). Spatial selection via feature-driven inhibition of distractor locations. Perception \& Psychophysics, 60, 727-746.

Cheal, M. L., \& Lyon, R. D. (1991). Central and peripheral precuing of forced-choice discrimination. Quarterly Journal of Experimental Psychology, 43A, 859-880.

Deutsch, J. A., \& Deutsch, D. (1963). Attention: Some theoretical considerations. Psvchological Review, 70, 80-90.

DunCaN, J. (1980). The locus of interference in the perception of simultaneous stimuli. Psychological Review, 87, 272-300.

DunCAN, J., \& HumphreYs, G. (1989). Visual search and stimulus similarity. Psychological Review, 96, 433-458.

Eriksen, C. W., \& Hoffman, J. E. (1972). Temporal and spatial characteristics of selective encoding from visual displays. Perception \& Psychophysics, 12, 201-204.

Folk, C. L., \& ANNETT, S. (1994). Do locally defined feature discontinuities capture attention? Perception \& Psvchophvsics, 56, 277-287.

Folk, C. L., \& Remington, R. W. (1996). When knowledge does not help: Limitations on the flexibility of attentional control. In A. F. Kramer. M. G. H. Coles, \& G. D. Logan (Eds.), Converging operutions in the study of visual selective attention (pp. 271-295). Washington, DC: American Psychological Association.

Folk, C. L., \& Remington. R. W. (1998). Selectivity in distraction by irrelevant featural singletons: Evidence for two forms of attentional capture. Journal of Experimental Psychology: Human Perception \& Performance, 24, 847-858.

Folk, C. L., Remington, R. W.. \& Johnston, J. C. (1992). Involuntary covert orienting is contingent on attentional control settings. Journal of Experimental Psychology: Human Perception \& Performance. 18. 1030-1044.

Folk, C. L., Remington, R. W., \& WRight, J. H. (1994). The structure of attentional control: Contingent attentional capture by apparent motion, abrupt onset, and color. Journal of Experimental Psychologr: Human Perception \& Performance, 20, 317-329.

Hillstrom, A. P., \& Yantis, S. (1994). Visual motion and attentional capture. Perception \& Psychophisics, 55, 399-411.

JONIDES, J. (1981). Voluntary versus automatic control over the mind's eye's movement. In J. [B.] Long \& A. [D.] Baddeley (Eds.), Attention and performance $I X$ (pp. 187-203). Hillsdale. NJ: Erlbaum.

JONIDES, J.. \& YANTIS, S. (1988). Uniqueness of abrupt visual onset in capturing attention. Perception \& Psychophysics, 43, 346-354.

KıM, M.-S., \& CAVE. K. R. (1995). Spatial attention in visual search for features and feature conjunctions. Psvchological Science, 6, 376-380.

KIM, M.-S.. \& CAVE, K. R. (1999). Perceptual grouping via spatial attention in a focused-attention rask. Manuscript submitted for publication.

KIM. M.-S.. \& CAVE, K. R. (in press). Grouping effects on spatial attention in visual search. Journal of General Psychology:

Koch, C., \& Ulıman, S. (1985). Shifts in selective visual attention: Towards the underlying neural circuitry. Human Neurobiologi, 4. 219-227.

Koshino, H., Warner. C. B., \& JUOl.A, J. F. ( 1992). Relative effectiveness of central, peripheral, and abrupt onset cues in visual search. Quarterly Journal of Experimental Psychology, 45A, 609-631.

Müller, H. J., \& RABbitT, P. M. A. (1989). Reflexive and voluntary orienting of visual attention: Time course of activation and resistance to interruption. Journal of Experimental Psychology: Human Perception \& Performance, 15, 315-330.

Nakayama, K., \& Mackeben, M. (1989). Sustained and transient components of focal visual attention. Vision Research, 29, 1631-1647.

Norman. D. A. (1968). Towards a theory of memory and attention. Psychological Review', 75, 522-536.

Pashler, H. (1988). Cross-dimensional interaction and texture segregation. Perception \& Psychophvsics, 43, 307-318.

POSNER, M. I. (1978). Chronometric exploration of mind. Hillsdale. NJ: Erlbaum.

POSNER. M. I. (1980). Orienting of attention. Quarterly Journal of Experimental Psychology, 32, 3-25.

Posner, M. I., SNyder, C. R. R., \& Davidson, B. J. (1980). Attention and the detection of signals. Journal of Experimental Psychology: General, 109. 160-174. 
Remington, R. W., Johnston, J. C., \& Yantis, S. (1992). Involuntary attentional capture by abrupt onsets. Perception \& Psychophysics, 51, 279-290.

SHIFFRIN, R., \& SCHNEIDER, W. (1977). Controlled and automatic human information processing: II. Perceptual learning, automatic attending, and a general theory. Psychological Review, 84, 127-190.

Theeuwes, J. (1991). Cross-dimensional perceptual selectivity. Perception \& Psychophysics, 50, 184-193.

Theeuwes, J. (1992). Perceptual selectivity for color and form. Perception \& Psychophysics, 51, 599-606.

THEeuwes, J. (1994). Stimulus-driven capture and attention set: Selective search for color and visual abrupt onsets. Journal of Experimental Psychology: Human Perception \& Performance, 20, 799-806.

THEeuwEs, J. (1995a). Perceptual selectivity for color and form: On the nature of the interference effect. In A. F. Kramer, M. G. H. Coles, \& G. D. Logan (Eds.), Converging operations in the study of visual selective attention (pp. 297-314). Washington, DC: American Psychological Association.

THEEUWES, J. (1995b). Temporal and spatial characteristics of preattentive and attentive processing. Visual Cognition, 2, 221-233.

TREISMAN, A. M., \& GELADE, G. (1980). A feature integration theory of attention. Cognitive Psychology, 12.97-136.

Trfisman, A. M., \& Sato. S. (1990). Conjunction search revisited. Jour- nal of Experimental Psychology: Human Perception \& Performance, 16, 456-478.

Wolfe, J. M., CAve, K. R., \& Franzel, S. (1989). Guided Search: An alternative to the feature integration model for visual search. Journal of Experimental Psychology: Human Perception \& Performance, 15, 419-433.

YANTIS, S. (1995). Attentional capture in vision. In A. F. Kramer, M. G. H. Coles, \& G. D. Logan (Eds.), Converging operations in the study of visual selective attention (pp. 45-76). Washington, DC: American Psychological Association.

YANTIS, S., \& EGETH, H. E. (1994). Visual salience and stimulus-driven attentional capture [Abstract]. Investigative Ophthalmology \& Visual Science, 35, 1619.

YANTIS, S., \& JoNiDES, J. (1984). Abrupt visual onsets and selective attention: Evidence from visual search. Journal of Experimental Psychology: Human Perception \& Performance, 10, 601-621.

YANTIS, S., \& JONIDES, J. (1990). Abrupt visual onsets and selective attention: Voluntary versus automatic allocation. Journal of Experimental Psychology: Human Perception \& Performance, 16, 121-134.

(Manuscript received May 27, 1997; revision accepted for publication June 18,1998 .) 